\title{
Multivariate analysis of X-ray diffraction and XAFS data
}

\author{
R. Caliandro', A. Mazzone', B. D. Belviso', Pietro Guccione², M. Milanesio ${ }^{3}$, L. Palin ${ }^{3}$, M. Lopresti ${ }^{3}$ \\ ${ }^{1}$ Institute of Crystallography, CNR, via Amendola 122/o, 70126, Italy, ${ }^{2}$ Dipartimento di Ingegneria Elettrica e dell'Informazione, \\ Politecnico di Bari, via Orabona 4, Bari, 70125, Italy, ${ }^{3}$ Dipartimento di Scienze e Innovazione Tecnologica, Università del Piemonte \\ Orientale, viale T. Michel 11, Alessandria, 15121, Italy.
}

rocco.caliandro@ic.cnr.it

The structural dynamics of chemical systems can be investigated by in situ or operando X-ray experiments. Advanced and fast computational methods are needed to cope with the huge amount of data collected, and to extract precious information hidden in data through a model-free analysis. Data analysis approaches based on multivariate analysis are particularly suited to this aim, as they are able to efficiently process in a probe-independent way multiple measurements, by considering them as a whole data matrix [1].

We have developed a fully automatic and big-data set of computing procedures based on principal component analysis, which is able to process with the same algorithms in situ/operando X-ray diffraction and XAFS data to extract qualitative and quantitative information. The multivariate approach has been adapted to treat crystallographic data, by optimizing the directions of the principal components [2], or by including kinetic models in the extraction of the reaction coordinate [3]. The procedure includes several preprocessing strategies that can be applied on crystallographic and XAFS data; among them a peak-shift correction to disentangling lattice variations from changes of the atomic parameters [4]. The procedures have been implemented in the computer program RootProf [5], available from www.ic.cnr.it/ic4/en/software/. It can be also used for fast on-site analysis while running in situ experiments (Fig.1).

Here we show how in situ experiments coupled with new data analysis methods can disclose the structural mechanism underlying: i) the thermal adsorption of gas in zeolites [4]; ii) the non-isothermal solid-state synthesis of materials based on poly-aromatic molecular complexes [6]; iii) the temperature-induced transitions of metal halide perovskites [7].
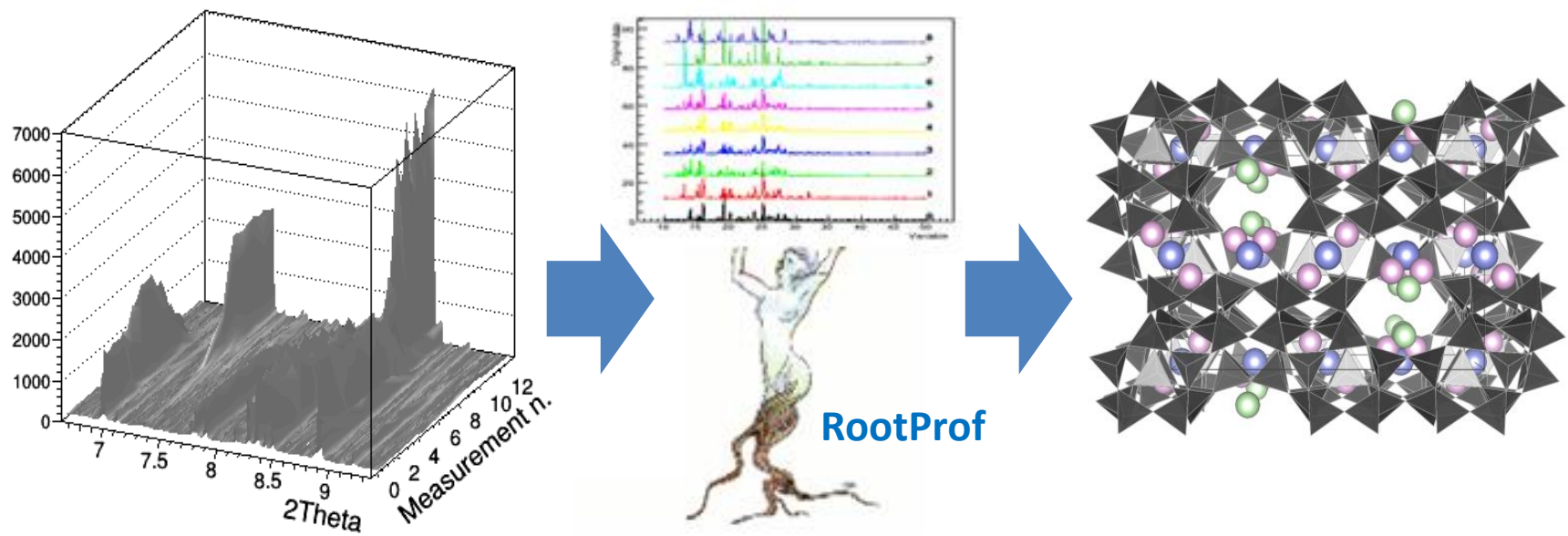

Figure 1. The RootProf program processes data from in situ experiments to locate atoms responding to an external stimulus.

[1] Guccione, P., Lopresti, M., Milanesio, M., Caliandro R. Crystals 11, 12.

[2] Caliandro, R., Guccione, P., Nico, G., Tutuncu, G., Hanson, J.C. (2015). J. Appl. Cryst. 48, 1679.

[3] Guccione, P., Palin, L., Belviso, B. D., Milanesio, M., Caliandro R. (2018). Phys. Chem. Chem. Phys. $20,19560$.

[4] Guccione, P. Palin, L., Milanesio, M., Belviso B.D., Caliandro, R. (2018). Phys. Chem. Chem. Phys. $20,2175$.

[5] Caliandro, R., Belviso, B. D., (2014). J. Appl. Cryst. 47, 1087.

[6] Palin, L., Conterosito, E., Caliandro, R., Boccaleri, E., Croce, G., Kumar, S., van Beek, W., Milanesio M. (2016). CrystEngComm 18, 5930.

[7] Caliandro, R., Altamura, D., Belviso, B. D., Rizzo, A., Masi, S., Giannini. C. (2019). J. Appl. Cryst. $52,1104$.

Keywords: Multivariate analysis; Principal component analysis; Qualitative analysis, Quantitative analysis.

Acta Cryst. (2021), A77, C599 